\title{
WiFi Access Point Deployment for Efficient Mobile Data Offloading
}

\author{
Eyuphan Bulut \\ Cisco Systems \\ 2200 President George Bush Highway \\ Richardson, TX 75082 \\ ebulut@cisco.com
}

\author{
Boleslaw K. Szymanski \\ Społeczna Akademia Nauk \\ ul Sienkiewicza 9, 90-113 Łódź, Poland, and \\ Rensselaer Polytechnic Institute \\ 110 8th St., Troy, NY 12180 \\ szymansk@cs.rpi.edu
}

Recently, there has been a tremendous increase in mobile data usage with the wide-spread proliferation of smartphone like devices. However, this increased demand from users has caused severe traffic overloading in cellular networks. Offloading the traffic through several other devices (femtocells, WiFi access points) have been considered to be immediate remedy for such a problem. Thus, in this paper, we study the deployment of WiFi access points (AP) in a metropoli$\tan$ area for efficient offloading of mobile data traffic. We analyze a large scale real user mobility traces and propose a deployment algorithm based on the density of user data request frequency. In simulations, we present offloading ratio that our algorithm can accomplish with different number of APs. The results demonstrate that our algorithm can achieve close to optimal offloading ratio that is higher than offloading ratios that existing algorithms can achieve with the same number of APs.

\section{Introduction}

Mobile data usage is rapidly growing due to increasing ubiquity of various mobile devices (i.e., smartphones, laptops) among users as well as the increasing demand of mobile data usage by each subscriber. People are using these devices for different activities such as web browsing, video/audio downloading and photo sharing. As Cisco recently announced in [1] that, global mobile data traffic will grow 18-fold from 2011 to 2016 , reaching 10.8 exabytes per month. About $71 \%$ of this traffic will be mobile video traffic. Also $4 \mathrm{G}$ connections will account for $36 \%$ of all mobile data traffic.

Having such mobile data explosion, it is inevitable that in the near future cellular networks will be overloaded and congested. Currently, this problem has already been experienced by subscribers of multiple operators in urban areas. Especially during the peak data usage times (rush hours etc.) breaks in the user calls occur due to the insufficient network bandwidth. Cellular operators need to provide solutions to this problem quickly because they can lose their subscribers feeling unsatisfied with the access quality and availability.

Recently, the network research community initiated some efforts to solve this challenging problem. Basically, there have been four types of solutions considered:

1. Increasing the number of radio base stations or selectively improving some of them [2].

2. Increasing network coverage through some small scale base stations (i.e. femtocells [3]) that are usually deployed at homes.

3. Upgrading cellular radio access technology to advanced next generation technologies (such as High Speed Packet Access (HSPA) and Long Term Evaluation (LTE)) to increase the bandwidth.

4. Utilizing WiFi networks for offloading the burden of cellular network $[4,5,6]$.

Even though all the above solutions can help in mitigating this problem, each one has unique advantages and disadvantages compared to the others. The first and the third solutions may require high financial investment and a long process of deployment. Moreover, they may bring low return gains especially with the current flat price policy. There have been some tiered pricing mechanisms adopted by operators in an attempt to slow the increase in demand but as the users are willing to pay even the high prices, tiered pricing solution will eventually become ineffective. Some providers even engaged in an equally futile attempt to educate their users about what each megabyte represents in terms of data and ask them for responsible and unwasteful access. 
Femtocells can be considered a good way offloading but they are somewhat new compared to WiFi APs which are already deployed in many places. They are deployed mostly for coverage extension but they can also provide indoor offloading. They operate on the same licensed spectrum as macrocells of the cellular network, so they do not require special hardware support on mobile phones. Yet, they come with some disadvantages like one-time cost of buying a new device which carry the price ranging from $\$ 100$ to $\$ 200$. However, their use can still be considered a good offloading strategy as long as users are willing to pay for the necessary devices. Good surveys on femtocells and their deployment are presented in $[3,8]$.

In summary, utilizing WiFi networks for cellular traffic offloading currently seems to be the most promising solution ${ }^{1}$ that can offer an immediate remedy to this problem. There are many WiFi access points available at many user locations such as homes, shops and universities. In addition to them, operators can also deploy their own APs at the locations where the access demand is high. Since these devices operate in different spectrum than the base stations, they also do not cause interference. Moreover, the bandwidth offered by WiFi is much higher than the bandwidth of cellular access.

In this paper, we focus on the problem of WiFi access point (AP) deployment for efficient mobile data offloading. To this end, we analyze a city wide real user mobility traces and propose an efficient AP deployment. We measure how much offloading can be achieved with different numbers of APs. Moreover, we look at the offloading efficiency of proposed deployment for future data usage by network users. We also find the optimal deployment by modeling the problem as an Integer Linear Programming (ILP) (see [14]) problem and solving it using the IBM ILOG CPLEX software [15]. The results indicate that proposed greedy heuristic based algorithm produces results that are close to the optimal solution.

The rest of the paper is organized as follows. In Section II, we talk about related work. In Section III, we present the details of proposed greedy heuristic based algorithm. In Section IV, we model the problem as an ILP problem and solve it exactly using the IBM ILOG CPLEX package. In Section V, we give the details of our simulation setting and present the

\footnotetext{
${ }^{1}$ This is also in agreement with the results of a recent survey [7] done by WBA/ITM among the world wide operator leaders who think WiFi offloading will be the most significant strategy in managing mobile network volume and rank this solution higher than femtocell deployment and upgrade to HSPA and LTE technologies.
}

simulation results. Finally, we end up with conclusion in Section VI.

\section{Related Work}

There are some previous studies that focus on an interesting type of offloading strategy called delay tolerant offloading. The idea behind the applicability of such strategy is that there is a remarkable amount of mobile data content (such as photo uploads to Flickr [5]) which are uploaded by users much later than their generation time at the mobile devices. Thus, in such situations, user devices can delay the upload of this kind of data up to some threshold and offload them automatically to $\mathrm{WiFi}$ access points [11] once they get into the range of them. If the opportunity to connect to a $\mathrm{WiFi}$ access point does not arise before the end of tolerable delay duration, regular cellular connection is used for uploading. A similar approach is proposed in [2] but the offloading is suggested to be done via some top base stations through which most of the content of users are uploaded.

This type of delayed offloading strategy is further justified by growing popularity of DTN type message exchanges among users (via Bluetooth or WiFi connections). Such exchanges increase the chance of offloading in the given time constraint by routing the data towards users which contact with offloading regions more frequently than others. In [9], Han et. al propose to select a set of most active users (using social relations [10]) through which the maximum offloading of all user data traffic will happen. Similarly, in MixZones algorithm in [11], opportunistic exchanges between users together with caching the popular content at users is proposed to achieve more efficient offloading.

Even though this type of delayed offloading strategies can relieve the burden of cellular network, they cannot be applied to users requesting real time data downloading, which forms the biggest portion (80\%$90 \%$ ) of today's mobile data traffic. Thus, their effective overall impact on offloading might be small. Moreover they also raise several user related concerns, such as passing of other users' data via some popular user devices, causing extra power consumption at such devices without benefits to their users. These concerns can be addressed through some incentive mechanisms [12], but a complete solution together with methods to address its risks has to be provided.

In this paper, we focus on an offloading solution using WiFi access points (Figure 1). An important benefit of WiFi networks is that they operate on unli- 


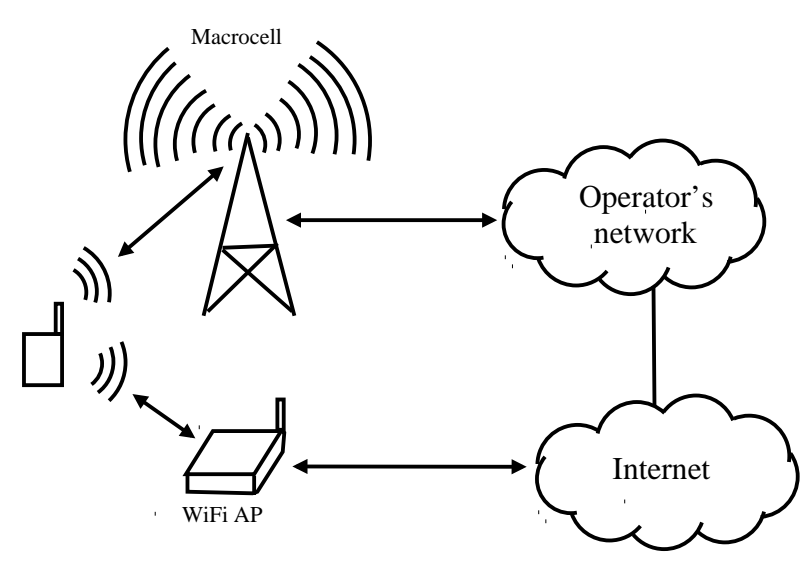

Figure 1: Mobile data offloading via Wifi APs

censed frequency bands, thus they do not cause interference with cellular networks. They also offer more bandwidth than what current cellular networks provide. Moreover, user mobile devices are already capable of communicating using WiFi. Capabilities of WiFi networks for traffic offloading have already been demonstrated in some studies like [5]. Moreover, operators have already been deploying their own WiFi APs to some points of interests (such as malls, markets etc.).

Despite the advances described above, the deployment of such WiFi APs has not been studied yet in large scale using real mobility traces. The works that are closest to the approach presented in this paper are [6] and [11]. They both propose algorithms for the deployment of WiFi APs for cellular traffic offloading. However, in [6], the AP locations are decided in a sequential manner without considering the efficiency of deployment. Similarly, the HotZones algorithm in [11] proposes to deploy APs to cover the areas of most used cell towers. Even though it deploys the APs to the area of most used macrocells (i.e. base stations), it does not consider the internal differences of subregions in the area of a macrocell in terms of user content generation density. A subregion inside a dense macrocell may not generate as much data as a subregion of another macrocell which is used less frequently. Moreover, the algorithm does not mention exactly how the APs are deployed in the areas of selected macrocells.

In this paper, we use fine grid cells in a city wide user area and propose to deploy the APs to the most dense grid cells in terms of user data request frequency using a greedy-based fast heuristic. We also show how close the results of this greedy approach are to the optimal solution. Moreover, in simulation sec-



Figure 2: Deployment with AP size grid cells

tion, we compare our algorithm with the algorithms presented in [6] and [11].

Apart from the AP deployment for offloading purposes, there are also some studies that propose algorithms for deploying APs for different reasons. For example, in [13], Liao et al. propose an algorithm to deploy minimum number of APs that provide full communication coverage while at the same time achieving the ability to locate a mobile device within a certain area no larger than a given accuracy parameter. Our work differs from such studies since we aim to do efficient offloading through a better deployment of APs.

\section{Greedy Heuristic Approach}

To increase the offloading efficiency, AP locations need to coincide with the popular mobile data request locations. For this reason, some operators have started to deploy WiFi APs in places like malls, markets and coffee shops where there might be a high population of their users. However, this type of deployment of APs to some limited number of user populated areas has to be extended to outdoor locations for a large scale offloading strategy.

Let's consider a city-wide area in several parts of which users often use their mobile devices to access Internet. Moreover, those users move around, thus, their locations and therefore the points from which the user data access requests come from change. Moreover, users sometimes turn their devices off. Also, they often use them for Internet access only during certain times. For example, it is expected to be more likely for people to use their phone while they are traveling in a taxi or using public transport rather than when they are driving themselves.

Having the mobility traces of people who are able to use mobile data, we need to deploy the APs where 
the presence of such users is high. Assuming that an AP can cover a circular range with radius $\mathrm{R}$, the question is how to deploy APs in such a way that the offloading is maximized and consequently the deployed APs are used efficiently.

Let $\Re=\left\{\left(r_{1}, w_{1}\right),\left(r_{2}, w_{2}\right), \ldots\left(r_{m}, w_{m}\right)\right\}$ be the list of pairs with each pair representing a location from which users make mobile data access requests and the corresponding weights of these accesses. Let $A P_{D}$ $=\left\{a_{1}, a_{2}, \ldots a_{K}\right\}$ be the candidate deployment of $K$ AP's (each with communication range of $R$ ), that is the list of locations in which WiFi APs are to be deployed. Let $I_{D}=\{i \in[1 . m] \mid \exists j \in[1, K]$ : $\left.\left|r_{i}-a_{j}\right| \leq R\right\}$ be the set of indexes of data access request locations covered by the candidate deployment $A P_{D}$. The goal is to maximize the following sum over all possible candidate deployments $\left(A P_{D}\right.$ 's):

$$
\sum_{i \in I_{D}} w_{i} r_{i}
$$

Since this problem is similar to maximum coverage problem, it is likely NP-hard. Hence, we propose here to use the greedy heuristic approach to solve it.

We start by dividing the entire region into equal size grid cells such that each cell represents the coverage of a single AP deployed at its center. Since an AP has an effective range of $R$, the side of each cell is equal to $\sqrt{2} R$, i.e. the side of the biggest square that will fit inside a circular AP range with radius $R$. Figure 2 illustrates the greedy approach. Then, we find, one by one, the most dense locations in terms of user mobile data request frequency denoting their locations by $m_{i}, m_{j}$.

Since this approach assumes that APs will only be deployed at the centers of grid cells, some most dense areas may be covered by neighbor cells partially. To mitigate this drawback, we propose an extended approach shown in Algorithm 1 in which AP centers are allowed to be in any corner of a cell in $n \times n$ sub-grid put over each grid cell representing an AP (see Figure 2). This extended version first divides the area into $S \times S$ grid and then further divides each of the resulting grid cells into $n \times n$ sub-grid. The algorithm then finds the possible AP location that gives the highest offloading ratio (i.e., which has the maximum data request frequency) under its coverage. Then, the other APs (with total AP count of $K$ ) are found similarly oneby-one, with each step removing the already covered areas by deployed APs. Here note that the disadvantage of introducing $n \times n$ subgrid is that the complexity of the algorithm increases as $n$ increases, but as we will show in simulation section, the advantage is that the results of heuristic get closer to the optimum.

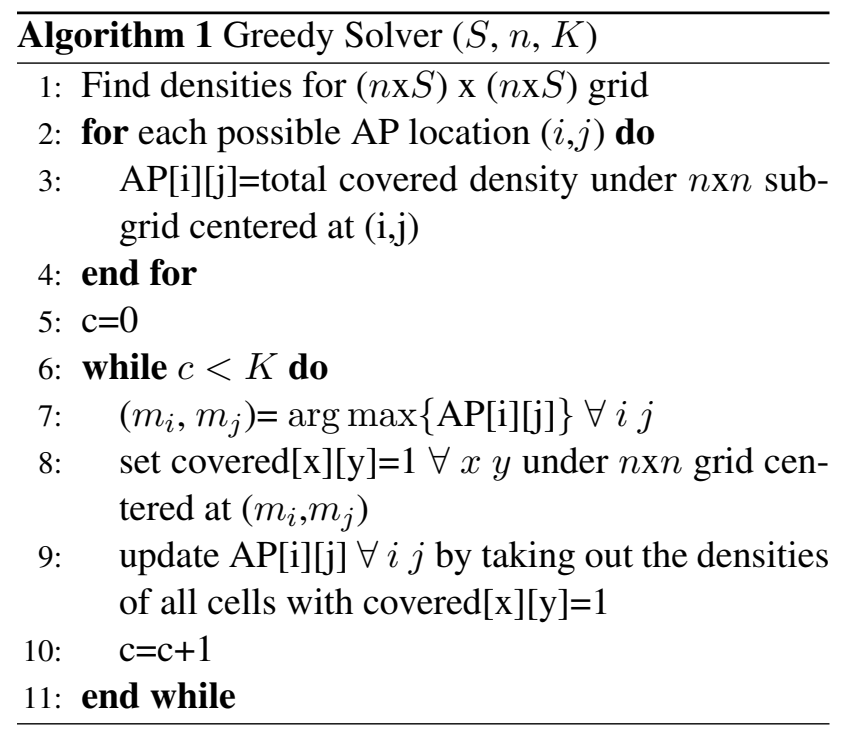

\section{ILP Solution}

To see how close the results of the greedy approach are to the optimum, we also formulate and solve exactly the problem of deploying APs using Integer Linear Programming (ILP) approach [14]. We first divided the entire area into very small cells and obtained an $N$ by $N$ grid. Then, representing an AP as an $n \times n$ small frame, we find the optimum deployment of $K$ APs that maximizes the offloading ratio.

In the ILP model, the goal is to find the optimal placement of $K$ small frames to grid locations, where placement of all small frames is defined by a pair of integer vectors $I=\left[i_{1}, \ldots, i_{K}\right]$ and $J=\left[j_{1}, \ldots, j_{K}\right]$, each of size $K$ such that for any $1 \leq k \leq K$ the pair $1 \leq i_{k} \leq N-n+1,1 \leq j_{k} \leq N-n+1$ defines the location of the North West corner of the small frame $k$. Let $I J$ denote the set of all possible vectors $I, J$ with their values integers from the interval $[1, N-n+$ $1]$. We used the following notation (in $x-y$ coordinate system):

$$
\begin{aligned}
S_{k, i, j} & = \begin{cases}1 & \text { if } i_{k} \leq i+n \text { and } j_{k} \leq j+n \\
0 & \text { otherwise }\end{cases} \\
y_{i, j} & = \begin{cases}1 & \text { if } \sum_{k=1}^{K} S_{k, i, j}>0 \\
0 & \text { otherwise }\end{cases} \\
w_{i, j} & =\text { weight (i.e., data request frequency)) of cell (i,j) }
\end{aligned}
$$

The binary variable $y_{i, j}$ indicates if cell $(i, j)$ is covered by any small frame. Given the above variables, the objective function is:

$$
\max _{I, J \in I J} \sum_{i=1}^{N} \sum_{j=1}^{N} w_{i, j} y_{i, j}
$$




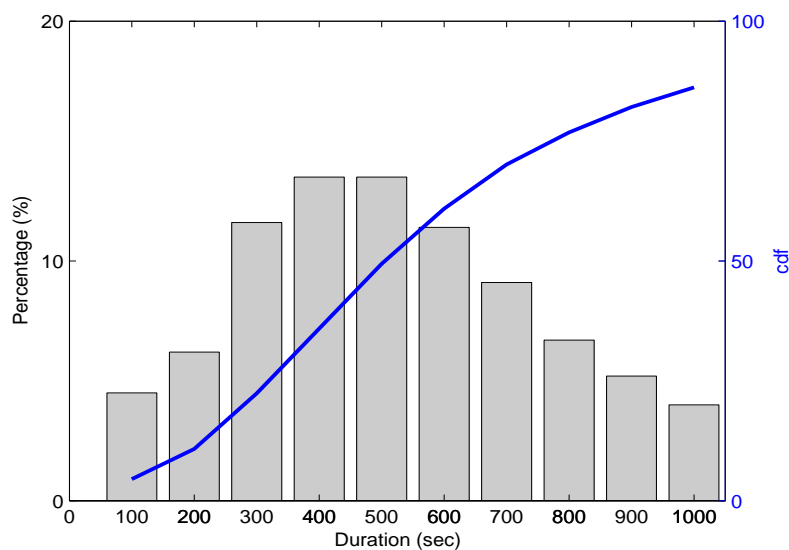

Figure 3: Distribution of occupancy durations.

Here, to compare optimal results with greedy results, we set $N=(n \times S)$ in simulations and solve it using IBM ILOG CPLEX [15] software.

\section{Simulations}

To evaluate the performance of proposed deployment strategy, we have built an event driven simulator that uses the real mobility traces. We used a taxi data set, which is publicly available [16], and determined the mobility of the users accordingly. The dataset consists of the traces of 536 taxis that operate in the city of San Francisco. For each taxi, the mobility trace records include the GPS location and the occupancy over a 30 day period. There are some discrepancies in the recording intervals. Thus, to make the evaluation with a data set that is less likely to include measurement errors, we pruned the dataset as in [6] by excluding the data about all taxis with the average sampling interval larger than $100 \mathrm{sec}$ and standard deviation bigger than $1000 \mathrm{sec}$. After this initial process, we obtained a set of data about 343 taxis. Moreover, we also extrapolated the locations of each of these taxis between the recorded two consecutive locations using available data.

Similar to [6], we generated the user data traffic (i.e., download requests) during the times taxis were occupied. This is justified by the fact that it is more reasonable to find a person using mobile devices (iPhone, iPad, vehicle's GPS etc.) to access Internet when traveling by taxi (as passenger) rather than in the case when driving a car or a taxi. We refer to a taxi occupancy duration as a journey. During each journey, we assume the user initiates a download request every $5 \mathrm{sec}$. If there is a WiFi access point located in the range of the location from which the download re- quest is initiated by the user, the download of the file is achieved via that $\mathrm{AP}^{2}$, offloading the corresponding traffic. Otherwise, download of the requested file is achieved through cellular network. In Figure 3, we show the distribution and the cumulative distribution function (cdf) of journey durations in all dataset. As the figure shows, the majority (about $86 \%$ ) of journey durations (and the corresponding sequence of download requests) last between 100 and $1000 \mathrm{sec}$, with an average of $612 \mathrm{sec}$. Considering that these journeys indeed are the taxi occupancy durations in San Francisco, the journey statistical characteristics seem reasonable.

We assume that at the beginning of the simulation there are no WiFi APs deployed in the area. Then, using each algorithm, we find the minimum number of APs that can guarantee the desired offloading ratio from cellular network. We compare our greedy algorithm and optimal results (from ILP solution) with the two previously introduced algorithms. In the first one [6], the AP locations are decided sequentially, one by one (we refer to it as "Sequential" in the discussion of results). That is, if a download request cannot be offloaded through a WiFi AP, the algorithm puts an AP there, and considers that the other download requests that fall in the range of this AP will also be offloaded through it. Then, the number of APs that will be deployed increases in this manner until allowed AP count is reached. The second algorithm is called HotZones [11] where APs are deployed to cover the areas of mostly used cell towers. Since, we simulate a user area which can be covered by several towers, we used four cell towers to get the results for this algorithm.

In outdoor environment, the effective $\mathrm{WiFi}$ range can vary from $5 \mathrm{~m}$ to $75 \mathrm{~m}$ [6]. Thus, for the greedy approach, we used two different AP ranges, $R(70 \mathrm{~m}$, $35 \mathrm{~m})$, yielding the side of a grid cell, $G_{s}, 100 \mathrm{~m}$ and $50 \mathrm{~m}$, respectively. The area we work on is $70 \mathrm{~km}$ by $70 \mathrm{~km}$, thus when $G_{s}=100 \mathrm{~m}, S=700$. Figure 4 and Figure 5 show the number of APs that needs to be deployed to achieve a given offloading ratio for all four algorithms with different grid sizes (so with different AP ranges). First of all, it is easy to observe that our greedy approach (without extension) provides results closer to the optimal solution than other algorithms. It can provide up to $13 \%$ and $24 \%$ higher offloading

\footnotetext{
${ }^{2}$ The download requests happening every $5 \mathrm{sec}$ during a journey can be considered as a request for downloading a small portion of a large file. Thus, currently, we assume that the small data portion of each download request can be downloaded within the time the user is in the range of AP. In future work, with continuous data access requests, we will study the impact of limited time of staying in the range of a WiFi AP on the results.
} 


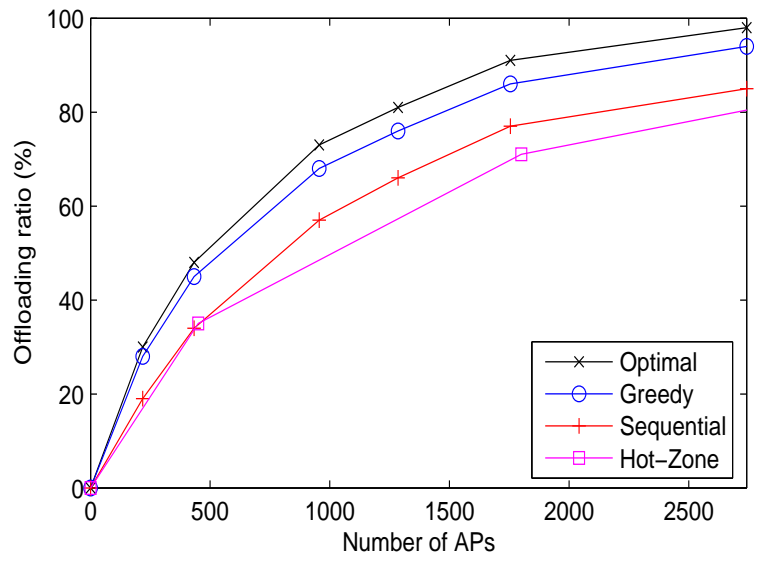

Figure 4: Number of APs vs. Offloading ratio when $G_{s}=100 \mathrm{~m}$.

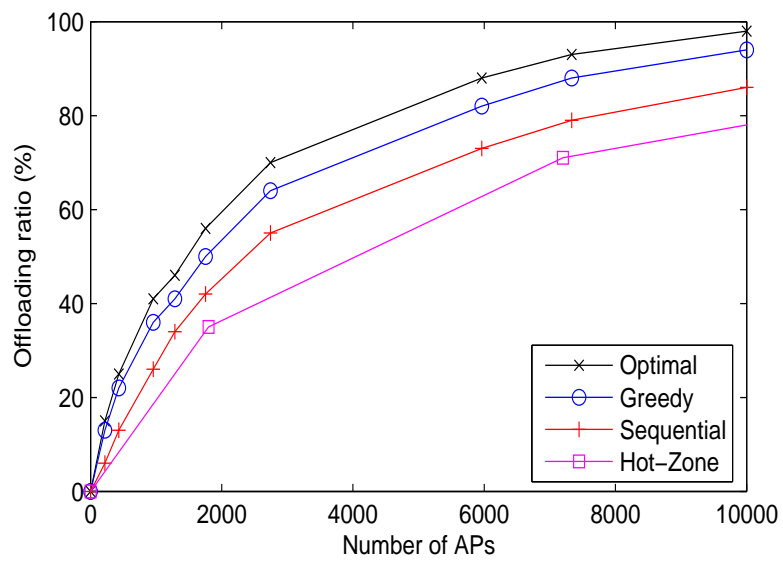

Figure 5: Number of APs vs. Offloading ratio when $G_{s}=50 \mathrm{~m}$.

ratio with the same AP count than the ratios achieved by the Sequential [6] and HotZones [11] algorithms, respectively. Moreover, looking at AP counts required to achieve a given offloading ratio shows that greedy algorithm sometimes needs only $65 \%$ and $45 \%$ of what Sequential and HotZones algorithms need, respectively. This clearly shows that greedy approach can provide the operators remarkable savings. Moreover, as Figure 6 shows, if the number of APs $(K)$ is small and we let the AP centers to be placed more arbitrarily within each grid cell, then greedy approach can give results much closer to optimal solution than the solution with placement of APs at the center of grid cells.

The above results consider the user data requests from all dataset and computes the offloading ratio if there were APs deployed in advance. To see the im-

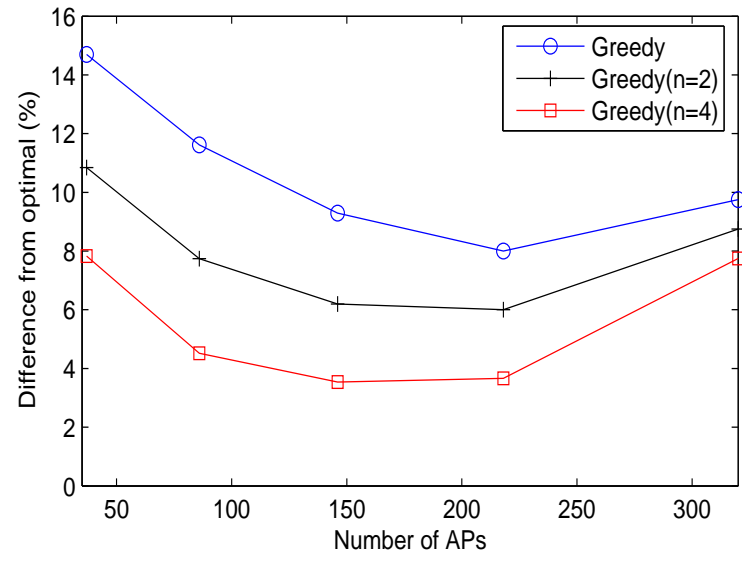

Figure 6: Results with greedy extensions.

pact of variance in the user behavior on the offloading strategy, as well as to see if this type of deployment works for future download requests, we also looked at the predictability of future node behavior and used only $x \%$ of the data ${ }^{3}$ to decide where to deploy the APs. Then, to measure the offloading ratio, we used the user requests that come from the rest of the data ${ }^{4}$. In Figure 7, we show the effect of the size of the training data used on the offloading ratio achieved by the heuristic. We used $25 \%, 50 \%$ and $100 \%$ of the data to find locations with the highest density of user demands for mobile bandwidth and deployed the APs accordingly. As the graph demonstrates, the offloading ratios achieved with deployment after using $25 \%$, $50 \%$ and $100 \%$ of data are very close to each other. This clearly shows that user behavior is more or less the same and deploying the APs to locations with the highest density of user requests for mobile data in greedy manner is a promising solution even for future mobile data offloading.

\section{Conclusion and Future Work}

In this paper, we studied WiFi access point (AP) deployment problem for efficient mobile data offloading. Analyzing user mobility traces, we proposed to deploy the APs to the locations with the highest density of user data access requests. We also found the optimal deployment by formulating the problem as an Integer Linear Programming problem and solving it using IBM ILOG CPLEX package. In simulation results, we showed that our algorithm achieves

\footnotetext{
${ }^{3}$ Since the processed data covers 576 hours of data after pruning, $\mathrm{x} \%$ of the data refers to the first 5.76x hours of user data.

${ }^{4}$ At $100 \%$ we used all data both for training and evaluation.
} 


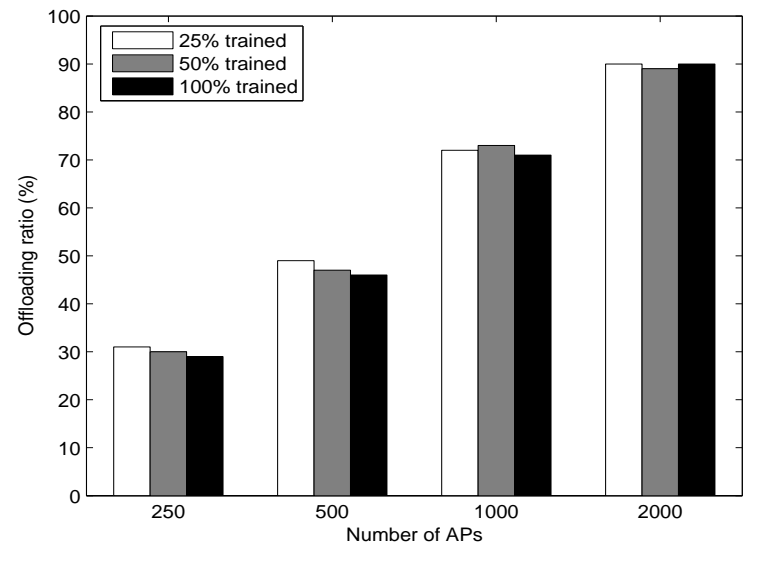

Figure 7: Effect of training data on offloading ratio of Greedy Algorithm $\left(G_{s}=100 \mathrm{~m}\right)$.

higher efficiency than efficiency yielded by the previous algorithms and gives results closer to optimal solution. Moreover, we demonstrated that our approach is also beneficial in offloading the future data requests of users.

In future work, we will take into account different parameters (different size of downloaded data, bandwidth limitations etc.) in our simulations. Moreover, we will work on market based incentive mechanisms [17] to motivate users to participate in offloading, so both users and operators can benefit together.

\section{Acknowledgment}

This research was sponsored by the US Army Research Laboratory and the UK Ministry of Defence and was accomplished under Agreement Number W911NF-06-3-0001. The views and conclusions contained in this document are those of the authors and should not be interpreted as representing the official policies, either expressed or implied, of the US Army Research Laboratory, the US Government, the UK Ministry of Defence, or the UK Government. The US and UK Governments are authorized to reproduce and distribute reprints for Government purposes notwithstanding any copyright notation hereon.

\section{References}

[1] Cisco Visual Networking Index, http://www.cisco.com/en/US/netsol/ns827/ networking_solutions_sub_solution.html, Feb, 2012.
[2] I. Trestian, S. Ranjan, A. Kuzmanovic, and A. Nucci, Taming the Mobile Data Deluge with Drop Zones, In IEEE/ACM Transactions on Networking, to appear, 2012.

[3] V. Chandrasekhar, J. G. Andrews, and A. Gatherer, Femtocell networks: A survey, IEEE Communications Magazine, vol. 46, no. 9, pp. 59-67, September 2008.

[4] A. Balasubramanian, R. Mahajan, and A. Venkataramani, Augmenting Mobile $3 G$ Using WiFi, in ACM MobiSys'10, Jun. 2010, pp. 209-222, 2010.

[5] K. Lee, I. Rhee, J. Lee, S. Chong, and Y. Yi, Mobile data offloading: how much can wifi deliver? in Proc. of Co-NEXT Š10, pp. 26:1-26:12, 2010.

[6] S. Dimatteo, P. Hui, B. Han and V. O. K. Li, Cellular Traffic Offloading through WiFi Networks, in Proc. of IEEE MASS, 2011.

[7] WBA Industry Report, Global developments in public WiFi, http://www.wballiance.com/ resource-centre/global-developments-wifireport.html, last accessed, May, 2012.

[8] J. G. Andrews, H. Claussen, M. Dohler, S. Rangan, M. C. Reed, Femtocells: Past, Present, and Future, IEEE Journal on Selected Areas in Communications, Vol. 30, No. 3, April 2012.

[9] B. Han, P. Hui,A. Kumar, M. V. Marathe, J. Shao, and A. Srinivasan, Mobile Data Offloading through Opportunistic Communications and Social Participation, IEEE Transactions on Mobile Computing, Vol. 11, No. 5, May 2012.

[10] P. Hui, J. Crowcroft, and E. Yoneki, Bubble rap: social based forwarding in delay tolerant networks, in Proc. of ACM MobiHoc, pp. 241-250, 2008.

[11] N. Ristanovic, J.-Y. L. Boudec, A. Chaintreau, V. Erramilli, Energy Efficient Offloading of $3 G$ Networks, in Proc. of IEEE MASS, 2011.

[12] X. Zhuo, W. Gao, G. Cao, and Y. Dai, WinCoupon: An Incentive Framework for $3 G$ Traffic Offloading, in Proc. of IEEE ICNP, 2011.

[13] L. Liao, W. Chen, C. Zhang, L. Zhang, D. Xuan, and W. Jia, Two Birds With One Stone: Wireless Access Point Deployment for Both Coverage and Localization, IEEE Transactions on Vehicular Technology, Vol. 60, No. 5, June 2011. 
[14] J. E. Beasley (editor), Advances in Linear and Integer Programming, Oxford Science, 1996.

[15] IBM ILOG CPLEX Optimizer, http://www01.ibm.com/software/integration/optimization/cplexoptimizer/, accessed on 07/04/2012.

[16] http://crawdad.cs.dartmouth.edu/meta.php?name= epfl/mobility, accessed on 07/04/2012.

[17] B. O. Holzbauer, E. Bulut and B. K. Szymanski, Socially-Aware Market Mechanism for Participatory Sensing, accepted to appear in Proc. of the First ACM International Workshop on Mission-Oriented Wireless Sensor Networking (MiSeNet) in conjunction with Mobicom, Istanbul, 2012. 